Journal of the Academy of Child and Adolescent Psychiatry, 37, $512-518$

M. D. Rudd Department of Psychology,

TexasTech University, Texas, USA.

Email: david.rudd@ttu.edu

doi: 10.II92/bjp.191.4.359

Author's reply: Professor Rudd raises important questions about whether it was appropriate to undertake this meta-analysis given the nature of interventions studied and the length of follow-up periods used. We believe that it can be appropriate to synthesis data from randomised trials to examine clinically important rare outcomes that individual studies are unlikely to be able to detect. For instance, psychosocial interventions for alcohol misuse are effective in reducing alcohol consumption but a range of factors, including clinical inertia, mean that they are not widely delivered. By synthesising data from trials conducted in a range of different settings, Cuijpers et al (2004) demonstrated that they are associated with a $30 \%$ reduction in subsequent mortality, a finding which may help to overcome some of the barriers to their delivery.

Although none of the studies we examined set out specifically to try to reduce suicide, it seems logical that interventions that are designed to reduce the incidence of suicidal behaviour should have an impact on the likelihood of fatal as well as non-fatal self-harm. Although several studies we included involved only brief interventions, such interventions have been shown to reduce the rate of suicide in other contexts, for instance in the period following discharge from in-patient psychiatric care (Motto \& Bostrom, 2001).

Most of the studies we included followed people for between 6 and 12 months after the initial episode of self-harm. Although this is a relatively short period it is also the period during which suicide is most likely to occur (Owens et al, 2002). By focusing on the period immediately following an episode of self-harm we maximised the likelihood of being able to demonstrate an impact on the rate of suicide.

However, we fully endorse Professor Rudd's comment that the results of our meta-analysis need to be interpreted with caution. Lack of data on suicide deaths in several of the trials that we identified meant that study power was limited. This resulted in wide confidence intervals around the pooled difference in suicide rates and it is therefore possible that additional psychosocial interventions do lead to reductions in subsequent suicide.

Cuijpers, P., Riper, H. \& Lemmers, L. (2004) The effects on mortality of brief interventions for problem drinking: a meta-analysis. Addiction, 99, 839-845.

Motto, J. A. \& Bostrom, A. G. (200I) A randomized controlled trial of postcrisis suicide prevention. Psychiatric Services, 52, 828-833.

Owens, D., Horrocks, J. \& House, A. (2002) Fata and non-fatal repetition of self-harm. Systematic review. British Journal of Psychiatry, 18I, 193-199.

M. J. Crawford Department of Psychologica Medicine, Imperial College London, UK. Email: m.crawford@imperial.ac.uk

O. Thomas Imperial College London, UK

N. Khan Central and North West London Mental Health NHS Trust, London, UK

E. Kulinskaya Imperial College London, UK doi: I0.II92/bjp.191.4.360

\section{Psychiatric disorder and looked after status}

Ford et al (2007) investigated the possible explanations for the increased prevalence of psychiatric disorder in children looked after by local authorities and linked looked after status with higher levels of psychopathology, educational difficulties and neurodevelopmental disorders. They suggested that services should bear in mind that a change of environment might be appropriate in providing help, at least in some cases.

After carefully reading the article, I think that Ford et al have missed an important aetiological factor: the influence of genetics. Studies (e.g. Howard et al, 2001) have shown that children of parents with mental disorder are likely to be looked after by another person or organisation. Biological factors which caused mental illness in the parents of children currently looked after by services might operate to cause the increased prevalence of psychiatric disorder in these children. Hence by neglecting the biological component of the bio-psychosocial model of mental illnesses, Ford $e t$ al have failed to provide a comprehensive assessment of causative factors in these children.

The authors could have included psychiatric disorder in the parents as a variable and divided the looked after group into children of parents with or without mental disorder. Ford et al have identified that neurodevelopmental disorders and learning difficulties are associated with increased prevalence of psychiatric disorder. Both are also associated with the future development of mental illnesses such as schizophrenia (Done et al, 1994; Lawrie et al, 2001) in which genetic factors play an important aetiological role (Cardno et al, 1999).

Cardno, A. G., Marshall, E. J., Coid, B., et al (1999) Heritability estimates for psychiatric disorders: the Maudsley twin psychosis series. Archives of Genero Psychiatry, 56, 162-168.

Done, D. J., Crow, T. J., Johnstone, E. C., et al (1994) Childhood antecedents of schizophrenia and affective illnesses: social adjustment at ages seven and eleven $B M$, 309, 699-703.

Ford, T., Vastanis, P., Meltzer, H., et al (2007)

Psychiatric disorder among British children looked after by local authorities: comparison with children living in private households. British Journal of Psychiatry, 190, 319-325.

Howard, L. M., Kumar, R. \& Thornicroft, G. (200I) Psychosocial characteristics and needs of mothers with psychotic disorders. British Journal of Psychiatry, I78, 427-432.

awrie, S. M., Byrne, M., Miller, P., et al (2001) Neurodevelopmental indices and the development of psychiatric symptoms in subjects at high risk of schizophrenia. British Journal of Psychiatry, 178, 524-530.

M. K. Sekar 176 Soho Hill, Handsworth, Birmingham BI9 IAG, UK.

Email: drmurali98@yahoo.com

doi: 10.II92/bjp.19I.4.360a

Authors' reply We totally agree with $\mathrm{Dr}$ Sekar's point that biological factors make an important aetiological contribution to the development of psychiatric disorder in children. We certainly did not intend to suggest that biological factors are any less important than psychological or social factors. Many childhood disorders are known to have a high level of heritability (Rutter $e t$ al, 2006). However, we should not forget that both our and previous studies suggest that similar risk factors operate in looked after children as in children living in private households, but that looked after children tend to have been exposed to more of them, sometimes at greater intensity (Stein et al, 1996; Ford et al, 2007). In our opinion, this includes biological as well as psychological and social factors.

Many studies have shown that parental psychiatric disorder is correlated with childhood psychiatric disorder (Rutter, 
2002). Although parental psychiatric disorder might increase the chance that children become looked after, this is not inevitable and there are many other reasons why children may enter the care system. In fact, only $6 \%$ of the children participating in the survey on which our analysis was based were accommodated primarily as a result of any type of parental illness.

As the survey involved no contact with the biological parents of participants and historical information about children who are looked after is notoriously scarce, we had no way of accurately assessing the mental health of the biological parents. Our paper refers to our frustration at the extremely limited amount of information available to the survey and in clinical practice, and we explicitly state that our analysis cannot be seen as covering all potential risk and resilience factors.

Even if we had access to data on the mental health of the biological parents, an excess of children with psychiatric disorder among parents with psychiatric disorder would not necessarily indicate a biological or genetic basis for this finding. The mean age that children participating in this survey entered the care system was between 7 and 8 years, and we know that mental illness can have an impact on parenting practices. Do the children of parents with mental illness have raised rates of psychiatric difficulties as a result of genetic vulnerability and/or exposure to maladaptive parenting, or perhaps both processes occur at the same time and/or moderate each other? The literature suggests that parenting is an important mediating variable, although other genetic and environmental factors also play a part in the familial aggregation of psychopathology (Ramachandani \& Stein, 2003). Crosssectional surveys are not able to disentangle such complex questions, as data about exposures and outcomes are gathered at the same time. Longitudinal designs would be needed to explore Dr Sekar's theory.

Ford, T., Vastanis, P., Meltzer, H., et al (2007)

Psychiatric disorder among British children looked after by local authorities: comparison with children living in private households. British Journal of Psychiatry, 190. 319-325.

Ramachandani, P. \& Stein, A. (2003) The impact of parental psychiatric disorder on children. BMJ, 327, 242243.

Rutter, M. (2002) Nature, nurture, and development: from evangelism through science to policy and practice. Child Development, 73, I-2I.
Rutter, M., Moffitt, T. E. \& Caspi, A. (2006) Geneenvironment interplay and psychopathology: multiple varieties but real effects. Journal of Child Psychology and Psychiatry, 47, 226-261.

Stein, E., Evans, B., Mazumber, R., et al (1996) The mental health of children in foster care: a comparison with community and clinical samples. Canadian Journal of Psychiatry, 4I, 385-39l.

T. Ford Institute of Psychiatry, King's College London, UK. Email: t.ford@iop.kcl.ac.uk

P. Vostanis Department of Health Sciences, Leicester University, UK

H. Meltzer, R. Goodman Institute of Psychiatry, King's College London, UK

doi: 10.1192/bjp.191.4.360b

\section{Lithium for prevention of Alzheimer's disease}

Nunes et al (2007) reported that the prevalence of Alzheimer's disease in a group of elderly patients with bipolar disorder who were on continuous lithium treatment was significantly less than in a similar group without recent lithium therapy. After controlling for age, lithium use remained associated with a smaller risk of Alzheimer's disease (age-adjusted OR $=0.079,95 \%$ CI 0.020-0.321). Conversely, Dunn et al (2005) showed that patients who received lithium had a significantly higher risk of dementia than those who did not (ageadjusted $\mathrm{OR}=1.8,95 \%$ CI 1.1-2.8).

Nunes et al (2007) found no differences between the lithium and the comparison group in neuropsychological performance after excluding patients with Alzheimer's disease. This is in accordance with our study using Mini-Mental State Examination (MMSE) scores (Terao et al, 2006). Our study, however, showed that patients with present and/or past history of lithium treatment had significantly better MMSE scores than patients without any history of lithium treatment (Terao et al, 2006). It is important to further investigate lithium in the prevention of Alzheimer's dementia with a large number of patients in prospective studies.

If lithium has a preventive effect for Alzheimer's disease, there may be two possible mechanisms. First, it might indirectly prevent dementia via its prophylactic effects on mood disorders, because the rate of dementia increased $13 \%$ with every episode leading to admission for patients with depressive disorder and $6 \%$ for patients with bipolar disorder, when adjusted for differences in age and gender (Kessing $\&$ Andersen, 2004). Second, lithium might directly prevent dementia via its inhibition of glycogen synthase kinase 3 (GSK-3) alpha (Phiel et al, 2003) and GSK-3 beta (Phiel \& Klein, 2001). Although Nunes et al (2007) found no significant differences in the number of previous depressive and manic episodes between the lithium and comparison groups, at present both possibilities should be borne in mind.

Dunn, N., Holmes, C. \& Mullee, M. (2005) Does lithium therapy protect against the onset of dementia? Alzheimer Disease and Associated Disorders, 19, 20-22.

Kessing, L.V. \& Andersen, P. K. (2004) Does the risk of developing dementia increase with the number of episodes in patients with depressive disorder and in patients with bipolar disorder? Journal of Neurology, Neurosurgery and Psychiatry, 75, 1662-1666.

Nunes, P. V., Forlenza, O. V. \& Gattaz, W. F. (2007) Lithium and risk for Alzheimer's disease in elderly patients with bipolar disorder. British Journal of Psychiatry, 190, 359-360.

Phiel, C. J. \& Klein, P. S. (200I) Molecular targets of lithium action. Annual Review of Pharmacology and Toxicology, 4I, 789-8I3.

Phiel, C. J., Wilson, C. A., Lee, V. M.Y., et al (2003) GSK-3 alpha regulates production of Alzheimer's disease amyloid-beta peptides. Nature, 423, 435-439.

Terao, T., Nakano, H., Inoue, Y., et al (2006)

Lithium and dementia: a preliminary study. Progress in Neuro-Psychopharmacology and Biological Psychiatry, 30, II25-II28.

T. Terao Oita University Faculty of Medicine, Oita, Japan. Email: terao@med.oita-u.ac.jp doi: 10.II92/bjp.19I.4.36I

Authors'reply: Dunn et al (2005) identified from the General Practice Research Database in the UK all cases of dementia between 1992 and $2002(n=9954)$ and compared the number of prescriptions of lithium for individuals with this diagnosis with a control group without dementia $(n=9374)$. They found that more patients with dementia $(n=47,0.47 \%)$ than controls $(n=40,0.43 \%)$ were exposed to lithium. We feel that this finding does not allow conclusions to be drawn as to whether lithium protects against or confers a risk for dementia, because it has been shown that patients with dementia have an increased risk of developing mania and depression (Nilsson et al, 2002) and are thus more likely to receive treatment, including lithium. Conversely, affective disorders themselves seem to increase the risk for dementia.

In a series of studies based on data from the Danish psychiatric case register, Kessing et al found that $14 \%$ of elderly patients with bipolar disorder and $16 \%$ with 